\title{
New tool in the box
}

\author{
Tong-Yi Zhang ${ }^{1,2}$ \\ ${ }^{1}$ School of Materials Science and Engineering, Harbin Institute of Technology, Shenzhen 518055, Guang Dong, China. \\ ${ }^{2}$ Materials Genome Institute, Shanghai University, Shanghai 200444, China.
}

Correspondence to: Prof. Tong-Yi Zhang, School of Materials Science and Engineering, Harbin Institute of Technology, Shenzhen 518055, Guang Dong, China. E-mail: zhangty@hit.edu.cn

How to cite this article: Zhang TY. New tool in the box. J Mater Inf 2021;1:1. http://dx.doi.org/10.20517/jmi.2021.01

Received: 2 Feb 2021 Accepted: 2 Feb 2021 Published: 3 Feb 2021

Academic Editor: Tong-Yi Zhang Copy Editor: Xi-Jun Chen Production Editor: Yue-Yue Zhang

Searching for new materials with desired functions and properties for targeted applications is always a key challenge in the field of material science and engineering. Although various advanced materials have been discovered in the past centuries, nowadays the material R\&D still depends primarily on the lengthy and painstaking trials inspired by scientific intuitions and experiences. This has increasingly become the bottleneck for the technological and social advancements.

The prominent "grand challenge" in materials science and engineering for the 21 st century is to invert the current paradigm in discovery, development and deployment of new materials from the present (deductive) cause-and-effect approach to a much more powerful and responsive (inductive) goal-means approach. This shift could substantially reduce time and cost in materials development and deployments. The Materials Genome Initiative (MGI) was launched in 2011 in the USA and the related projects have been launched worldwide, seeking to activate a paradigm shift in how materials discovery, development and deployment could be accelerated, through a tighter integration of theory, experiment, computation, and artificial intelligence. Great achievements have been and will continue to be accomplished in MGI.

In contrast to the computation- and experiment-driven modes whose essence is either the deduction of known physical laws or factual judgment, the data-driven mode uses artificial intelligence (AI)-based methods such as machine learning to reveal the relationships embedded in data and to predict candidate materials, also known as materials informatics. Materials informatics aims to combine traditionally bioinformatics with computational methodologies for supporting more efficient research through identifying strategies for time and cost-effective analysis. Clearly, this "new tool in the box", namely data + $\mathrm{AI}$, will definitely revolutionize the existing routines of material research. It is also important to point out

\footnotetext{
(c)

(C) The Author(s) 2021. Open Access This article is licensed under a Creative Commons Attribution 4.0 International License (https://creativecommons.org/licenses/by/4.0/), which permits unrestricted use, sharing, adaptation, distribution and reproduction in any medium or format, for any purpose, even commercially, as long as you give appropriate credit to the original author(s) and the source, provide a link to the Creative Commons license, and indicate if changes were made.
}

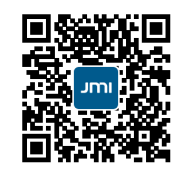


that the data-driven mode will in no wise replace the other two modes. Instead, it is a powerful extension and upgrade of conventional cognitive paradigms.

Data are the basic prerequisite for the full implementation of the materials informatics. With the progress of computing science and experimental techniques, an enormous quantity of material data is being generated around the world, but only a drop in the ocean is used in individual material analyses considering the diversity and complexity of material problems as well as data standardization and data sharing. Material scientists are longing for ways of solving material problems especially inverse problems through materials informatics, which stimulates the interdisciplinary collaborations between experts from materials science and computer science.

However, material data are not always big. In many materials areas, the quantity of data is quite limited because of costly and time-consuming experiments. How can machine learn small data and gain knowledge is a great challenge in materials informatics. This may require that the domain knowledge of material scientists plays more important roles in data mining (including developing new algorithms) conducted by computer scientists.

We expect that the joint effort of domain knowledge (materials science) and machine learning (computer science) will definitely speed up the materials $\mathrm{R} \& \mathrm{D}$, as well as the progress of computer science in the near future. Although materials informatics is pretty much in its infancy and the full power of its synergy is still far from being fully realized, it has showcased game-changing potential, and a wealth of opportunities exist in this arena. The impact of their synergistic interaction is expected to evolve quickly in the near future through building on the rapid evolution of the broader machine learning ecosystem, thereby providing the opportunity for transformative advances to the discovery, design, and deployment of new materials.

We firmly believe that the new Journal of Materials Informatics, as a truly interdisciplinary publication, will contribute to the advancement of materials genome and informatics as a new tool in the box of materials $\mathrm{R} \& \mathrm{D}$, and serve as the footstone for the future development of this interdisciplinary field.

\section{DECLARATIONS}

\section{Authors' contributions}

The author contributed solely to the article.

\section{Availability of data and materials}

Not applicable.

\section{Financial support and sponsorship}

None.

\section{Conflicts of interest}

The author declared that there are no conflicts of interest.

\section{Ethical approval and consent to participate}

Not applicable.

\section{Consent for publication}

Not applicable.

\section{Copyright}

(c) The Author(s) 2021. 